\title{
Application of Block-chain Technology in the Development of Functional Agriculture
}

Yang Luo

School of Economics and Management, Zhejiang Ocean University, Zhoushan, Zhejiang 316022

Author:

Luo Yang (October 1988), male, from Lichuan, Hubei, Tujia, Master's degree, mainly engaged in regional economy, e-commerce research

Absrtact: with the continuous growth of national economic income and the gradual improvement of health consciousness, the public not only put forward higher requirements for the type, quality and safety of agricultural products, but also began to pay more attention to the health function of agricultural products. Therefore, on the basis of ensuring the supply of high quality and safe agricultural products, through the development of ecological high value agriculture, further planting and cultivating agricultural products with full health care function has become the development direction of agriculture in the future.However, due to the influence of soil conditions, climate environment, management reputation and other factors, the asymmetric information hidden behind the current functional agriculture in China directly affects the healthy development of functional agriculture. Applying block chain technology to the development of functional agriculture can not only innovate the new mode of agricultural economic development, but also effectively eliminate all kinds of obstacles to development under information asymmetry, thus accelerating the development of functional agriculture.

Keywords: blockchain technology; functional agriculture; economic model; development exploration

After the publication of New Directions of Cryptograph» in 1976, the new cryptography began to enter the public view and laid the foundation for the development of block chain. Combined with tree data structure, elliptic encryption algorithm, POW algorithm, hash function and other technologies and methods to support the birth of block chain technology. With the global financial crisis of 2008 and the simultaneous crisis of trust between the two parties, Satoshi Nakamoto posted the bitcoin white paper Bitcoin : APeer-to-Peer Electronic Cash System》 on P2P foundation website $^{[1]}$ Since the advent of Bitcoin, blockchain technology, as the underlying core technology of Bitcoin, has gradually been paid attention to in various fields in the world. Wang Lu, Liu Shuangyin, Zhang Lei et al .(2019) believe that block chain is a chain structure, which is a decentralized public account book technology formed by combining data blocks in chronological order and combining with cryptography, consensus mechanism, intelligent contract and other technologies ${ }^{[2]}$ By means of Citespace and other tools, Fang Yi and Meng Jing found that China's achievements in blockchain basic technology, application scenarios, security analysis and evaluation have increased exponentially ${ }^{[3]}$ Agriculture: Hong Tao (2016) has done research on the application of blockchain in the field of agricultural products e-commerce in China ${ }^{[4]}$ Wang Xiaochun (2018) carried out upstream exploration of agricultural products under block chain technology ${ }^{[4]}$ Cheng Chuanxing (2019) tries to embed block chains to restrain breaking and manage the agricultural industry chain ${ }^{[6]}$ However, the blockchain technology has not been applied to functional agriculture to explore and solve a series of problems.

Copyright $\odot 2020$ Yang Luo

doi: $10.18282 /$ le.v9i6.1334

This is an open-access article distributed under the terms of the Creative Commons Attribution Non-Commercial License

(http://creativecommons.org/licenses/by-nc/4.0/), which permits unrestricted non-commercial use, distribution, and reproduction in any medium, provided the original work is properly cited. 
The concept of functional agriculture is the first to appear in the "Road Map for the Development of Agricultural Science and Technology in China to 2050", which was jointly completed by the Strategic Research Group in the Field of Agriculture of the Chinese Academy of Sciences from October 2007 to March $2009{ }^{[7]}$ The simple definition is to produce agricultural products with health function through agriculture. In September 2019, the State Development and Reform Commission and the State Grain and Material Reserve Bureau, in their guidance for speeding up the construction of a modern grain industry system, clearly stressed the need to increase the effective supply of specialized varieties and functional foods, guide grain processing to extend to medicine and health care, and continuously improve the added value and comprehensive benefits of products. ${ }^{[8]}$ This provides policy support for the further development of functional agriculture. In the process of the development of functional agriculture, how to eliminate the psychological obstacles between the supply and demand of functional agricultural products under information asymmetry and prevent opportunism is a problem worth exploring. From the individual point of view, three meals a day in daily life usually means that the human brain has a hunger consciousness, which promotes the human body to produce eating demand. This hunger is dominant and disappears with the degree of eating. As for whether the food we eat can meet the needs of the human body for various nutrients is recessive, which is the relationship between "full" and "eat well" as the saying goes. In general, we can divide our agriculture into four stages of development according to the utility analysis of agricultural products, namely, original agriculture, high-yield agriculture, organic agriculture and functional agriculture. They all show different emphases in pursuing agricultural product utility and social utility (Table 1).

Table 1: Analysis of Agricultural Development Stage and Its Utility in China

\begin{tabular}{|c|l|}
\hline Development phase & \multicolumn{1}{c|}{ Utility analysis } \\
\hline Original Agriculture & $\begin{array}{l}\text { Inheriting traditional agricultural varieties is conducive to stabilizing agricultural production and re- } \\
\text { ducing the risk of farmers farming agricultural products. }\end{array}$ \\
\hline High-yield agriculture & Pursue grain production increase, raise unit area output value, solve "full" problem. \\
\hline Organic agriculture & $\begin{array}{l}\text { Reduce environmental damage, rational use of resources, achieve sustainable development, and } \\
\text { solve the problem of safe and reliable agricultural products. }\end{array}$ \\
\hline Functional agriculture & $\begin{array}{l}\text { On the basis of high yield organic, adjust the internal nutrient composition of agricultural products } \\
\text { reasonably and solve the problem of "eating well ". }\end{array}$ \\
\hline
\end{tabular}

It can be seen that functional agriculture is the same agricultural system in our country, which is in line with the actual development needs of contemporary agriculture, is conducive to adjusting the agricultural system structure of our country, improving the nutrition of agricultural products, and enhancing the market core competitiveness of agricultural products.

\section{Bottleneck of 1. Functional Agricultural Industry Development}

\subsection{Resources and environment constraints functional agriculture development}

On the one hand, the vast soil structure of our country is rich and diverse, and the contents of various elements are different, which leads to the limitation of regional resources in the development of functional agriculture. On the other hand, in the soil rich in scarce elements, because in the agricultural high-yield agricultural stage in order to pursue shortterm production efficiency unscientific use of pesticides, fertilizers, resulting in a large number of harmful elements residue. In such an environment, it is not ensured that the cultivated agricultural products contain scarce trace elements, and whether other harmful elements or heavy metals in agricultural products exceed the standard.

\subsection{Unsincerity affects the circulation of functional agricultural products}

Annotating the value of healthy functional agricultural products is often $30 \%$ higher than that of ordinary agricultural products $\sim 50 \%$. If the price of agricultural products is doubled several times, this is the key to promote agricultural economic growth. But in the real life transaction, there are many substandard, false real phenomenon. If this kind of economic phenomenon can not be limited controlled, according to Greshin's law, there will be the phenomenon of bad money expelling good money, which reduces the audience's recognition of functional agricultural products and slows down the development of functional agriculture in China.

\subsection{Incomplete functional standards}

By changing the dietary structure of edible functional agricultural products, the intake of reasonable trace elements is 
beneficial to health. But functional agricultural products are not omnipotent agricultural products, we should objectively understand and identify their functions. Because the standard of function appraisal is not perfect, many producers and operators deliberately exaggerate their product utility and even use it as a tool to obtain excess profits, which not only greatly increases the unstable factors of society, but also violates the original intention of the development of functional agriculture.

\section{Block chain Technology Support Mechanism for Functional Agriculture}

\subsection{Consensus-counting and regulation of full-function agricultural geographical resources}

The development speed of functional agriculture is limited by the local soil, climate, environment and other conditions. The development of functional agriculture according to local conditions, and the realization of low cost and high output is in line with the fundamental competition requirements of agricultural economy. Based on "Internet + " and big data analysis, the content of trace elements in soil structure of each region is proved by consensus algorithm, and then the soil distribution information block containing trace elements is formed and imported into the whole block chain. On the one hand, we can judge whether the corresponding origin has the basis of functional agricultural production according to the information on the block chain, so as to further guide farmers to plant corresponding functional agricultural products and avoid unnecessary consumption of human, material and financial resources. On the other hand, the disclosure of regional information is also conducive to the construction of large functional agricultural group production base, shorten the time of enterprise research, can quickly form regional advantages and create functional agricultural products industry brand.

\subsection{Smart contracts achieve trade balance for functional agricultural products}

The decentralization and unforgeability of blockchain technology can realize the restriction in the circulation link and form Nash equilibrium in each circulation channel. Suppose a functional agricultural product Q (the proposed function of the product has no difference),In the process of entering the market circulation, there are $n$ distributors, and each dealer is in the marketing strategy $G=\left\{\mathrm{S}_{1}, \mathrm{~S}_{2}, \mathrm{~S}_{3}, \cdots, \mathrm{S}_{\mathrm{n}}, \mathrm{u}_{1}, \mathrm{u}_{2}, \mathrm{u}_{3}, \cdots, \mathrm{u}_{\mathrm{n}}\right\} ;$ Then the strategy combination formed

$S_{n}^{*-}\left\{S_{1}^{*}, S_{2}^{*}, S_{3}^{*}, \cdots, S_{n}^{*}\right\}$, Will exist on the blockchain, and any $i$ will satisfy $u_{1}\left(S_{1}^{*}, S_{s}^{*}\right) \geq u_{j}\left(S_{1}^{*}, S_{s}^{*}\right)$. This comes from the active implementation of the smart contract of blockchain technology, that is, the parties will actively abide by this agreement, and there is no Pareto improvement. In this way, the benefits of agricultural production can be maximized, and circulators can also use smart contracts to reduce risky transactions in the business process.

\subsection{Unsymmetrical encryption facilitates the disclosure of functional information}

The concealment and asymmetry of information are the important reasons for buyers' skepticism about functional agricultural products. The core pursued by the consumers of functional agricultural products is precisely the "function ". The asymmetric encryption of data under block chain technology can master the whole process data of production, processing, inspection, circulation and sale of functional agricultural products from the source, so that the data collected and recorded can ensure its accuracy. With the symmetrical grasp of information by consumers, the trace element content of the product is really understood. And in the actual circulation process, through the technical principle of block chain combined with the design of breach of contract system, the use of good faith to limit the operation can achieve the standardization of circulation links, thus eliminating dishonest business behavior.

\section{Approach to implement blockchain technology in 3. functional agriculture}

\subsection{Focus on applied talent training}

The application of block chain technology in functional agriculture is based on network information. The main body of talent application technology, block chain technology has been developed rapidly in recent years, but in the face of agricultural operators are not very familiar, let alone reasonable use. Agricultural practitioners themselves have a low level of education, in 2017 rural personnel structure received high school education only 12.3, college above 1.5. In order to ensure the combination of block chain technology and functional agriculture, it is necessary to increase the training of talents and let the operators of agriculture understand the specific application methods on the basis of familiarity. The government should increase investment in the training of corresponding talents, guide agricultural 
colleges and universities and other vocational education institutions, and set up corresponding courses in a targeted manner. At the same time, it can also carry out technical propaganda and study in village groups. Let talent training work in the forefront of the use of technology.

\subsection{Improvement of laws and regulations}

The block chain technology based on information network depends on the social integrity system to a great extent. In order to be able to develop steadily for a long time, we must have perfect laws, regulations and systems. For example, in the process of intelligent contract performance, there is a deliberate imagination of breach of contract, there must be a corresponding punishment mechanism, in order to protect the reasonable operation of the technology. To use illegal means to deliberately attack the system and seek improper interests, we should crack down strictly according to the law, and create a clean and upright operating environment.

\subsection{Accelerating technological innovation}

Block chain technology is a subversive technological progress, is bound to be actively used in all fields. When the volume is more and more, we will pay attention to the optimization and upgrading of our own technology. To prevent $51 \%$ attack, to actively realize cross-training technology, to make more numerical resources accessible and shared, to ensure that personal privacy is not leaked, to adopt a more active consensus mechanism and to improve the operation speed of the block chain itself. In the process of continuously improving many technologies, various fields, including functional agriculture, will also continue to develop, serving development according to technology, developing ways to support technological innovation, and forming a spiral structure between technological and economic development. So that both sides continue to progress forward.

\section{Summarize and Discuss the Significance of Developing Functional Agriculture}

Functional agriculture is the development direction of agriculture in the future and the new motive force to promote the development of agricultural economy. Its benign development is helpful to the transformation and upgrading of the whole agricultural system, which is of great significance. After analyzing the problems in the development of functional agriculture, the block chain technology is introduced, which attempts to eliminate the corresponding influencing factors through the consensus algorithm of block chain, intelligent contract, asymmetric encryption and data block technology, and then further combines the present situation of agricultural countryside in China. This paper briefly describes the implementation of block chain technology in functional agriculture. Its fundamental purpose is to promote the continuous development of China's agricultural economy, thus improving the level of the entire national economy.

\section{References}

1. Satoshi Nakamoto S.Bitcoin : A peer-to-peer electronic cash system[J]. White paper,2008.

2. Wang Lu, Liu Shuangyin, Zhang Lei, Xu Longqin, Cao Liang. A Review of Block-chain Technology [J].][5] Digital Communications World ,2019(08):134-135.

3. Fang Yi, Meng Jing, Wang Lili, Tang Yan, Wang Lei. Application of Block-chain Technology in Agriculture in China [J].] by Document Measurement China Science and Technology Information ,2019(14):102-104.

4. Hong Tao. Application of Block chain in the Field of Agricultural E-commerce in China [J].].5 China market ,2016(09):65-68.

5. Wang Xiaochun. Exploration of Agricultural products uplink under Block chain Technology [J] big data era 2018(03):24-26

6. Fu Hao; Zhao Cuiping; Cheng Chuanxing. Block chain embedding, constraint breaking and governance of agricultural industrial chain [J].]; and Agricultural economy 2019(11)

7. Zhao Qiguo, Yin Xuebin. Agriculture of Our Future [J].] Functional Agriculture Journal (Natural Science Edition)2017,37(7):457

8. the State Development and Reform Commission, the State Grain and material Reserve Bureau, and the guidance on speeding up the construction of a modern grain industry system with the goal of high quality development (National Grain (2019)240). 2019-09-18. 